\title{
A Comparative Study on Green Wall Systems
}

This paper has been retracted at the request of the editor of the

American Journal of Civil Engineering and Architecture.

The editor retracts this paper due to duplicate publication. 